\title{
miR-132 Regulates Dendritic Spine Structure by Direct Targeting of Matrix Metalloproteinase 9 mRNA
}

\author{
Magdalena Jasińska ${ }^{1,2,3}$. Jacek Milek ${ }^{1,2,4}$ - Iwona A. Cymerman ${ }^{5}$ - Szymon Lęski ${ }^{6}$. \\ Leszek Kaczmarek $^{1}$ - Magdalena Dziembowska ${ }^{1,2}$
}

Received: 27 March 2015 / Accepted: 10 August 2015 /Published online: 29 August 2015

(C) The Author(s) 2015. This article is published with open access at Springerlink.com

\begin{abstract}
Mir-132 is a neuronal activity-regulated microRNA that controls the morphology of dendritic spines and neuronal transmission. Similar activities have recently been attributed to matrix metalloproteinase-9 (MMP-9), an extrasynaptic protease. In the present study, we provide evidence that miR-132 directly regulates MMP-9 mRNA in neurons to modulate synaptic plasticity. With the use of luciferase reporter system, we show that miR-132 binds to the 3'UTR of MMP-9 mRNA to regulate its expression in neurons. The overexpression of miR-132 in neurons reduces the level of endogenous MMP9 protein secretion. In synaptoneurosomes, metabotropic glutamate receptor (mGluR)-induced signaling stimulates the dissociation of miR-132 from polyribosomal fractions and shifts it towards the messenger ribonucleoprotein (mRNP)-containing fraction. Furthermore, we demonstrate that the overexpression of miR-132 in the cultured hippocampal neurons
\end{abstract}

Leszek Kaczmarek

1.kaczmarek@nencki.gov.pl

Magdalena Dziembowska

m.dziembowska@nencki.gov.pl; m.dziembowska@cent.uw.edu.pl

1 Laboratory of Neurobiology, The Nencki Institute, Pasteura 3, 02-093 Warsaw, Poland

2 Laboratory of Molecular Basis of Synaptic Plasticity, Center of New Technologies, University of Warsaw, Warsaw, Poland

3 School of Molecular Medicine, Żwirki i Wigury 61, 02-091 Warsaw, Poland

4 Institute of Biochemistry and Biophysics, 02-106 Warsaw, Poland

5 Laboratory of Molecular and Cellular Neurobiology, The International Institute of Molecular and Cell Biology, Warsaw, Poland

6 Laboratory of Neuroinformatics, The Nencki Institute, Pasteura 3, 02-093 Warsaw, Poland from Fmr1 KO mice that have increased synaptic MMP-9 level provokes enlargement of the dendritic spine heads, a process previously implicated in enhanced synaptic plasticity. We propose that activity-dependent miR-132 regulates structural plasticity of dendritic spines through matrix metalloproteinase 9.

Keywords Matrix metalloproteinase 9 (MMP-9) · miR-132 . Structural plasticity of dendritic spines

\section{Introduction}

Reorganization of the neuronal networks supports physiological phenomena of learning and memory, as well as major neuropsychiatric pathologies, such as epilepsy, addiction, schizophrenia, to name just a few. The excitatory synapses located at the dendritic spines (small membranous protrusions extending from the dendrites) are the fundamental structural units of this circuitry that can be modulated in response to neuronal activity. Recently, microRNAs have emerged as important regulators of molecular events occurring at the synapses, and being responsible for their plastic changes.

MicroRNAs (miRNAs) are small, $\sim 21$-nt-long RNAs that post-transcriptionally regulate gene expression in eukaryotes. In animals, miRNAs bind to partially complementary sites in mRNAs, leading to translational repression and mRNA deadenylation and degradation [1-3]. Many studies confirmed the presence of microRNAs in the dendrites and synapses where they are believed to fine-tune the local expression of synaptic proteins [4]. Mir-132 is a neuronal activity-regulated microRNA, which expression is induced by plasticityimplicated transcription factor CREB - cAMP response element-binding protein [5]. MiR-132 was shown to be rapidly upregulated in model conditions of the synaptic plasticity, 
e.g., in the primary visual cortex after eye opening [6] and conversely, downregulated by mononuclear deprivation [7]. The expression levels of the primary and precursor forms of miR-212 and miR-132 were induced during long-term potentiation (LTP; an electrophysiological model of the synaptic plasticity) in the rat adult dentate gyrus [8] and upon brainderived neurotrophic factor (BDNF) stimulation of primary cortical mouse neurons [9]. MiR-132 is present in the dendrites and at synapses [10-12], what makes it a potential unique regulator of locally translated synaptic proteins. Interestingly, the miR-132 knockout (resulting in miR-132/ miR-212 double knockout, as they are coded by the same gene) is not essential for development or fertility of mice; however, its function is related to specific aspects of synaptic plasticity [13]. During the development of hippocampal neurons, miR-132 promotes dendritic arborization and neurite outgrowth [14-17]. In mature neurons, the effect of miR132 is mostly related to the changes in spine morphology and synaptic transmission. [13]. When ectopically expressed in hippocampal neurons, miR-132 induces enlargement of dendritic spines $[18,6]$.

Matrix metalloproteinase-9 (MMP-9), an endopeptidase that regulates the pericellular environment through cleavage of its protein components, plays a critical role in the regulation of dendritic spine morphology and synaptic plasticity $[19,20]$. Recently discovered local translation of MMP-9 mRNA at synapses in response to neuronal stimulation may provide major insight into mechanisms involving MMP-9 in the synaptic plasticity [21]. Furthermore, MMP-9 local translation was found to be regulated by fragile $\mathrm{X}$ mental retardation protein (FMRP). Mutations in its gene result in the most common form of inherited human mental retardation linked to autistic symptoms (fragile X syndrome, FXS), and respective knockout (Fmr1 KO) mice provide excellent animal model to approach the human condition. Recently, we demonstrated that in Fmr1 KO mice, the local, synaptic translation of MMP-9 mRNA is upregulated, what results in the higher MMP-9 activity at the synapse [22]. Locally secreted MMP9 is involved in the reorganization of spine architecture [23, 24] and was shown to regulate spine morphology in Fmrl KO mice [25]. One of the characteristic features of FXS neurons is the presence of immature, long, and thin dendritic spines [26, 27]. Interestingly, the genetic elimination of MMP-9 in Fmr1 KO mice by creating a double Fmr1/ MMP-9 knockout mice rescued the aberrant phenotype of Fmr1 KO dendritic spines, ameliorated the abnormal mGluR5-dependent LTD, as well as aberrant behaviors [28]. Moreover, the reduction of exaggerated MMP9 mRNA translation in Fmrl(-/y) mice by the inhibition of translation initiation (reduction of eIF4E phosphorylation) also rescued core behavioral deficits of this mice as well as synaptic plasticity alterations and dendritic spine morphology defects [29]. These data show that the level of MMP-9 expression at the synapses is important for the maintenance of their proper structure and function.

Thus, both, miR-132 and MMP-9 have been implicated in the regulation of structural plasticity in neurons. However, no direct link between those important molecules has been revealed as yet. In the present study, we provide evidence for miR-132-dependent regulation of MMP-9 mRNA in neurons that result in structural changes of dendritic spines.

\section{Methods}

\section{Animals}

We have used early adult P26-P45 Fmr1 KO mice on FVB background as well as their wild-type littermates. Prior to the experiment, the animals were kept in the laboratory animal facility with free access to food and water with a $12 \mathrm{~h} \mathrm{light/}$ dark cycle. All the procedures with animals were carried out according to guidelines of the First Warsaw Ethical Committee on animal research with appropriate permissions.

\section{Preparation of Synaptoneurosomes}

Synaptoneurosomes were prepared from early adult P26-P45 wild-type and Fmr1 knockout mice by differential filtration as described previously [22]. Briefly, cortex and hippocampi from one mouse were dissected and homogenized on ice in $1 \mathrm{ml}$ of homogenization buffer (containing (in $\mathrm{mM}$ ) $125 \mathrm{NaCl}$, 1.2 MgSO4, 2.5 CaCl2, 1.53 KH2PO4, 212.7 glucose, 4 $\mathrm{NaHCO} 3, \mathrm{pH} 7.4$ set with carbogen), supplemented with protease inhibitor cocktail (Sigma-Aldrich), and $100 \mathrm{U} / \mathrm{ml}$ mammalian placental RNase inhibitor (Fermentas). The final volume of the homogenate was set at $10 \mathrm{ml}$ per one brain with homogenization buffer, and the samples were passed through a series of nylon mesh filters consecutively, 100, 60, 30, and $10 \mu \mathrm{m}$ (Millipore Bedford, MA) and centrifuged at $1000 \times \mathrm{g}$ for $15 \mathrm{~min}$. The pellets containing synaptoneurosome preparations were washed once in the same volume of homogenization buffer, centrifuged as before, and resuspended in homogenization buffer to a final protein concentration of $1 \mathrm{mg} / \mathrm{ml}$. Protein concentration was measured using BCA protein assay (Pierce).

\section{RNA Co-Immunoprecipitation and qRT-PCR}

The immunoprecipitation with the anti-FMRP antibody (7G1 from Developmental Studies Hybridoma Bank) was performed as described previously [22]. Freshly prepared synaptoneurosomes were resuspended in $1200 \mu$ l of precipitation buffer (10 mM HEPES, pH 7.4, $400 \mathrm{mM} \mathrm{NaCl}, 30 \mathrm{mM}$ EDTA, and $0.5 \%$ Triton X-100) with protease inhibitor cocktail (Sigma-Aldrich) and $100 \mathrm{U} / \mathrm{ml}$ RiboLock (Fermentas). 
First samples were precleared with $120 \mu 1$ of Dynabeads Protein A (Life Technologies) for $2.5 \mathrm{~h}$. After preclearing, $100 \mu \mathrm{l}$ of each supernatant was saved as an input fraction for Western blot to test procedure efficiency. Next, samples were precipitated overnight in $4{ }^{\circ} \mathrm{C}$ with $120 \mu \mathrm{l}$ of antibodybound Dynabeads Protein A, with either anti-FMRP antibody or normal mouse IgG. Total RNA was extracted with TRIreagent (Sigma). For RNA isolated after FMRP-immunoprecipitation, the LSM mRNA (from Arabidopsis thaliana) was added into the reverse transcription reaction mix (6 pg per reaction) as the external control. Next, the RNA was reverse transcribed using random hexamer primers (Fermentas). Realtime PCR was performed using SYBRGreen PCR Master Mix (Applied Biosystems) and the following primers:

PSD95 (F:TGAGCTATGAGACGGTGACG, R:CGC TTAGGACGTGTCGTATG),

LSM (F: TCTTCTCTCTCCGTGTCCA, R:TGATCA ATTCGCCAATGCG),

MMP-9 mRNA quantification after immunoprecipitation was performed using TaqMan PCR Master Mix (Applied Biosystems) and TaqMan primer/probe set for MMP-9 (Applied Biosystems, Mm00442991_m1). The quantification of miR-132 was carried out by using TaqMan MicroRNA Assay (Applied Biosystems). Values were calculated according to the ddCT method, using LSM mRNA as an external control.

\section{DNA Vectors}

For the luciferase assays, the reporter constructs containing the coding sequence of firefly luciferase (FF-luc) and the untranslated 3'UTR sequence of rat MMP-9 mRNA under the synapsin 1 promoter (pSyn-Luc-3'UTR-MMP9) were prepared. pSyn-Luc-3'UTR-MMP9-MUT construct was obtained by site-directed mutagenesis of four nucleotides within the putative biding site for miR-132 in the pSyn-Luc-3'UTRMMP9 plasmid.

The perfect seed match sequences for miR-132 or miR-9 were cloned downstream of the firefly luciferase in the FF-luc expressing vector. Constructs were prepared using the following primers:

miR132 seed match (L:CCCGACCATGGCTGTAGAC T G T TA G GC GC G C C C G A C C AT G GCTGTA GACTGTTAGG, R:CCTAACAGTCTACAGCCA TGGTCGGGCGCGCCTAACA GTCTACAGC CATGGTCGGG)

miR9 seed match (L:CCTCATACAGCTAGATA ACCAAAGAGGCGCGCCTCATACA GCTAGATAACC AAAGAGG, R:CCTCTTTGGTTATCTAGCTGTAT GAGGCGCGCCT CTTTGGTTATCTAGCTGTATGAGG)

MMP9-MUT (L:CGCTGTCCTTTCTTGTTGG CAGTTTTCTAATAAACACGGATCC, R:GGATCCG TGTTTATTAGAAAACTGCCAACAAGAAAG GACAGCG).
The control vector expressing Renilla luciferase p-RLCMV (Promega) was used for cotransfection and normalization of the results. pEZX-MR04 plasmid (GeneCopoeia) was used for the overexpression of miR-132.

\section{Primary Neuronal Cultures and Transfection}

We have used different neuronal primary cell cultures for specific transfection protocols.

(i) For the efficient electroporation, freshly isolated and dissociated neurons from the P1 rat cortices were used. The optimized protocol allows for the $50 \%$ of transfection efficiency. Cortical cultures prepared from $\mathrm{P} 0$ rat brains were used for luciferase assays. Cells were transfected after dissection and dissociation using Rat Neuron Nucleofector ${ }^{\circledR}$ Kit (Lonza) according to corresponding Amaxa ${ }^{\mathrm{TM}}$ Optimized Protocol. After transfection, neurons were cultured in Neurobasal-A medium (Life Technologies) supplemented with B27 (Life Technologies), glutamine, glutamate, and penicillin-streptomycin. Luciferase activity was analyzed 48-72 $\mathrm{h}$ after transfection.

(ii) For the transfection of differentiated rat cortical neurons at DIV7, we used NeuroMag Transfection Reagent (Ozbiosciences). We wanted to obtain sufficient transfection efficiency to perform the luciferase assay. The cells were transfected with pSyn-Luc-3'UTR-MMP9 reporter vector at DIV7 and incubated for additional 7 days. At 14 DIV, we quiescent spontaneous neuronal activity by inhibiting voltage-gated sodium channels (TTX), AMPA/ kainate receptors (CNQX), NMDA receptors (APV), and L-type calcium channels (nifedipine-all from SigmaAldrich) for $10 \mathrm{~h}$. After the lysis, the luciferase activity was analyzed in the silenced and untreated cells.

(iii) To asses the effect of miR-132 on the morphology of dendritic spines in the mature neurons, we have used primary hippocampal cultures (DIV14) obtained from Fmr1 $\mathrm{KO}$ and wt mice. The hippocampi were obtained from embryonic (E18) murine brains, dissociated and grown in neurobasal medium (Life Technologies) supplemented with B27 (Life Technologies), glutamine, glutamate, and penicillin-streptomycin. Neurons were transfected at DIV14 with vectors for miR-132 or EGFP overexpression using Lipofectamine ${ }^{\circledR} 2000$ (Life Technologies). Cells were fixed $72 \mathrm{~h}$ after transfection (4\% paraformaldehyde $/ 4 \%$ sucrose in PBS) and immunostained with antiGFP antibody (MAB3580 from Millipore).

\section{HEK293 Cell Culture and Transfection}

HEK293 cells were cotransfected with reporter plasmids using polyethylenimine (PEI) (Sigma-Aldrich) and cultured 
in DMEM+glutamax medium (Life Technologies) supplemented with $10 \%$ FBS and penicillin-streptomycin.

\section{Luciferase Assay}

Luciferase activity was quantified $48 \mathrm{~h}$ after transfection using Dual-Glo ${ }^{\circledR}$ Luciferase Assay System (Promega) according to the manufacturer's protocol. Relative expression of reporter constructs was determined by normalizing the ratio of FF-luc to RR-luc activity.

\section{Polyribosome Fractionation, Isolation of RNA, and Quantification}

Synaptoneurosomes prepared from wt and Fmr1 KO mice were stimulated with $50 \mu \mathrm{M}$ DHPG (Tocris Bioscience) for $15 \mathrm{~min}$, as described previously [22]. Synaptoneurosomes were lysed using a buffer $(20 \mathrm{mM}$ Tris- $\mathrm{HCl} \mathrm{pH} 7.4$, $125 \mathrm{mM} \mathrm{NaCl}, 5 \mathrm{mM} \mathrm{MgCl}_{2}$, protease, and RNase inhibitors) containing $1.5 \%$ Triton-X100, and membranous structures were removed by spinning at $20,000 \times \mathrm{g}$ for $40 \mathrm{~min}$. Resulting supernatant was loaded on a $10-50 \%$ linear sucrose gradient (prepared in $20 \mathrm{mM}$ Tris- $\mathrm{HCl} \mathrm{pH} \mathrm{7.5,} 125 \mathrm{mM} \mathrm{NaCl}$, and $5 \mathrm{mM} \mathrm{MgCl} 2$ ) and spun at 38,000 rpm for $2 \mathrm{~h}$ in SW41 rotor. Each gradient was separated into five fractions. Total RNA was isolated from each of the polysomal fractions using Tri-Reagent (Sigma) according to the manufacturer's instruction. RNA was reverse transcribed using SuperScript III Reverse Transcriptase (Invitrogen) and random hexamer primers (Fermentas). Reverse transcription and qPCR of miR-132 was performed using TaqMan MicroRNA Assay (Applied Biosystems).

\section{Gel Zymography}

Medium from cortical neurons transfected by electroporation with plasmids overexpressing miR-132 or EGFP was collected $36 \mathrm{~h}$ after transfection. The protein concentration was measured using the BSA kit (Pierce), and the equal concentration of the protein was mixed with $2 \times$ sample buffer Tris-Glycine SDS (Novex). Samples were subjected to electrophoresis under nondenaturing, nonreducing conditions in SDS-PAGE Tris-glycine $8 \%$ acrylamide gels containing $0.5 \%$ gelatin (POCH). Next, gels were washed twice for $20 \mathrm{~min}$ in $2.5 \%$ Triton X-100 and incubated for $72 \mathrm{~h}$ in the zymography buffer (50 mM Tris, $\mathrm{pH} 7.5,10 \mathrm{mM} \mathrm{CaCl}_{2}, 1 \mu \mathrm{M} \mathrm{ZnCl}_{2}, 1 \%$ Triton $\mathrm{X}-100)$ at $37{ }^{\circ} \mathrm{C}$. After incubation, gels were stained with $0.5 \%$ Coomasie. The intensity of white bands on the blue background corresponding to the MMP-9 and matrix metalloproteinase 2 (MMP-2) activity was quantified with ImageJ program. The relative activity of MMP-9 was normalized to the MMP-2 activity that was constant in the analyzed samples.

\section{Western Blotting}

Primary rat cortical neurons were transfected by electroporation with plasmids overexpressing miR-132 or EGFP. Cells were lysed $36 \mathrm{~h}$ after transfection in the $1 \times$ SDS sample buffer, denatured and fractionated on $10 \%$ SDSpolyacrylamide gels. The samples were electrotransferred onto PVDF membranes (Immobilon-P, Millipore), which were blocked $2 \mathrm{~h}$ at room temperature with $10 \%$ nonfat milk in PBST. After blocking, the membranes were incubated at $4{ }^{\circ} \mathrm{C}$ overnight with anti-MMP-9 antibody (\#3852, Cell Signaling) or anti-beta-actin (\#A1978 Sigma) all diluted in $5 \%$ nonfat milk PBST. Membranes were incubated $1 \mathrm{~h}$ at room temperature with peroxidase-labeled secondary antibody in $5 \%$ nonfat milk in PBST and visualized with ECLplus reagent (GE Healthcare). The levels of MMP-9 and beta-actin were quantified using ImageJ software for three independent experiments. MMP-9 level was normalized to beta-actin.

To test the immunoprecipitation efficiency, Western blot with anti-FMRP antibody (\#7104; Cell Signaling) was performed.

\section{Spine Clustering and Spine Parameters Estimation}

Images of fixed, immunofluorescently stained neurons and dendritic spines were captured with LEICA TCS SP8 SMD confocal system. Spine area, length, and width were estimated using SpineMagick software [30]. Spine length was calculated as the length of the path from spine top to the dendrite along the virtual skeleton of a spine. For the purpose of shape analysis, the images of individual spines (obtained by semiautomatic segmentation) were first straightened, that is, transformed so that the virtual skeleton of each spine formed a straight line. Next, the images were rescaled to normalize the spine area, and for each spine we defined $d(h)$, diameter of the spine as a function of distance from the dendrite. To classify the spines according to shape, we used a two-step procedure: first, all $6558 \mathrm{~d}(\mathrm{~h})$ functions were clustered into 36 clusters; next, the clusters were manually sorted into three groups (mushroom, stubby, and thin spines) based on average images of the clusters and visual inspection of spines comprising the clusters.

To measure widths and lengths of necks and heads of mushroom spines, we approximated each normalized image with stereotyped shapes: a ball representing the spine head, a cylinder representing the spine neck, and a cone representing the part of a spine closest to the dendrite. Dimensions of fitted elements were then taken as dimensions of head and neck. Data analysis was performed using custom scripts written in Python, using NumPy and SciPy [31, 32] and Matplotlib [33]. 


\section{Results}

\section{MMP-9 mRNA and miR-132 Associate with FMRP in Synaptoneurosomes}

miR-132, as well as several other miRNAs, is associated with FMRP in mouse brain [18]. FMRP can also bind MMP-9 mRNA [22]. miR-132 and MMP-9 mRNA were not studied together in the context of FMRP; however, these findings suggested that they could be a part of the same protein-RNA complex. To answer this question, we have performed the RNA co-immunoprecipitation following the protocol used for the identification of MMP-9 mRNA as a target of FMRP with the anti-FMRP (7G1-1) antibody using protein extracts from synaptoneurosomes isolated from cerebral cortex and hippocampi of wt and Fmr1 KO mice. As shown in Fig. 1a, FMRP was precipitated with the 7G1-1 FMRP antibody in synaptoneurosomal extracts prepared from wild-type brains, while it was not present in the Fmr1 KO immunoprecipitates (IPs). To measure the amount of mature miR-132 and MMP-9 mRNA that coimmunoprecipitated with anti-FMRP antibody, we performed quantitative polymerase chain reaction (qPCR). As shown in Fig. 1b, c, both MMP-9 mRNA and miR-132 were significantly enriched in the wild-type FMRP precipitates when compared to the Fmr1 KO synaptoneurosomes, suggesting indirectly the interaction between those two RNAs. PSD-95 mRNA, a known target of FMRP [34], was also present in the FMRP complex (Fig. 1d). Moreover, we have checked the level of miR-132 expression in the hippocampi of wt and Fmr1 KO mice and we have not observed statistically significant differences in the level of miR132 expression between the two genotypes, suggesting that basal miR-132 expression is not affected by the lack of FMRP protein.

\section{miR-132 Targets 3'UTR of MMP-9 mRNA and Regulates the Level of MMP-9 Protein in Neurons}

In silico analysis of the murine MMP-9 mRNA sequence revealed the presence of putative miR-132 binding site within the 3'UTR of the transcript (Fig. 2a). Furthermore, the binding region complementary to the miR-132 was found to be conserved between species. To determine whether miR-132 binds to the 3' UTR of MMP-9 mRNA, we used the luciferase reporter assay. The coding sequence of firefly luciferase (FFluc) was fused with the 3'UTR of MMP-9 mRNA, and, to ensure the efficient expression in neurons, luciferase was cloned under the control of synapsin 1 promoter. The expression of mature miR-132 from the commercial vector ( $\mathrm{pEZX}$ MR04 from Gene Copoeia) was validated on the FF-luc reporter constructs containing perfect-match miRNA targetsites for miR-132 and miR-9 (sensors). As shown in Fig. 2b, miR-132 specifically downregulates the expression of the miR-132 seed containing sensor but not the one containing the miR-9 sequence. The reporter construct was then cotransfected with the miR-132 expressing vector to the HEK293 cells together with a plasmid encoding Renilla reniformis luciferase (RR-luc), used for normalization. We

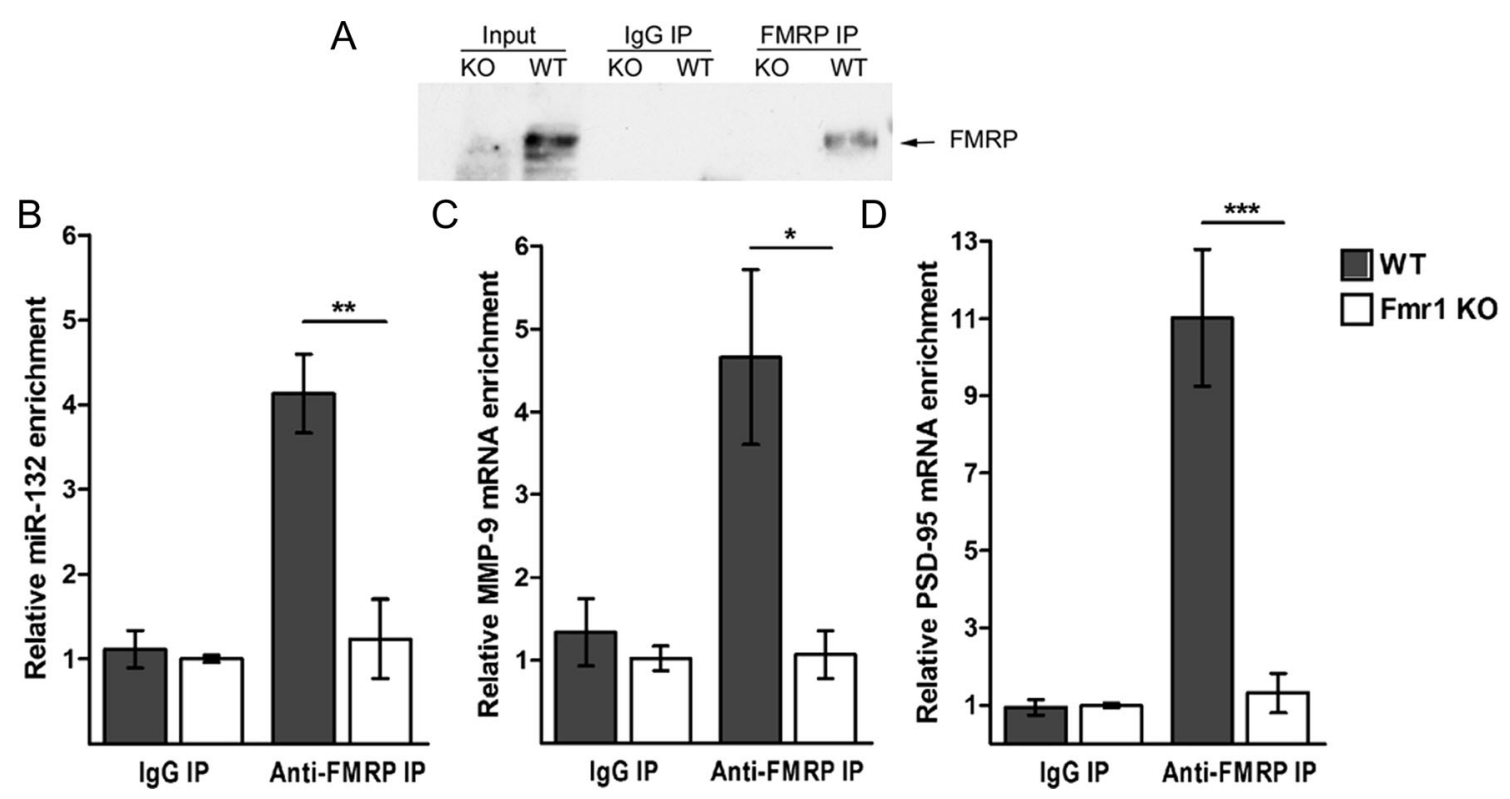

Fig. 1 MMP-9 mRNA and miR-132 associate with FMRP in synaptoneurosomes. a MMP-9 and miR-132 recovery from FMRPimmunoprecipitation of wt and Fmr1 KO mice. Western blot analysis of the immunoprecipitated FMRP from mouse synaptoneurosomes shows FMRP precipitated by the anti-FMRP 7G1-1 antibody in wt mice. Fmr1 $\mathrm{KO}$ extracts as well as IgG IPs were used as negative controls. b-d RT-
qPCR analysis of RNAs immunoprecipitated by anti-FMRP antibody - $\mathbf{b}$ miR-132, c MMP-9, and d PSD-95 (positive control) RNAs detected in the wt over Fmr1 KO immunoprecipitates. Values were normalized to the external "spike" control gene. Error bars indicate SEM, $n=3,{ }^{*} p<0.03$; $*^{*} p<0.01 ; * * * p<0.001$ by Student's $t$ test 


\section{A}

$\begin{array}{ll}\text { Rno-miR-132 } & \text { 3' GCUGGUACCGAC-AUCUGACAAU 5' } \\ \text { MMP-9 3'UTR } & \mathbf{2 2 7 5 | 5} \text { ' TGTCCTTTCTTGTTGGACTGTTT 3'|2297 }\end{array}$

B

\begin{tabular}{|c|c|c|}
\hline $\begin{array}{r}\text { Synapsin } 1 \\
\text { promoter }\end{array}$ & FF luciferase & $\begin{array}{c}\text { miR-132 } \\
\text { perfect seed match }\end{array}$ \\
\hline $\begin{array}{l}\text { Synapsin } 1 \\
\text { promoter }\end{array}$ & FF luciferase & rfect seed $n$ \\
\hline
\end{tabular}

C
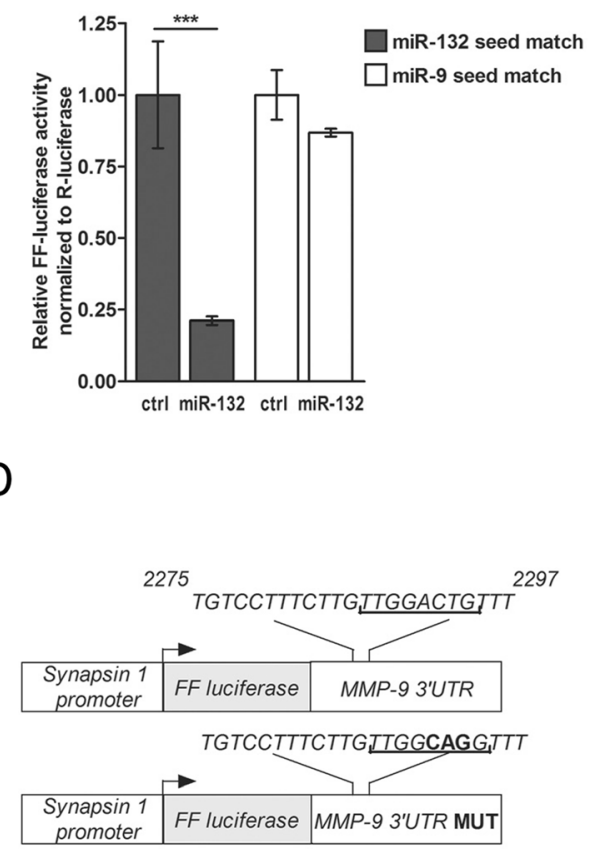

E
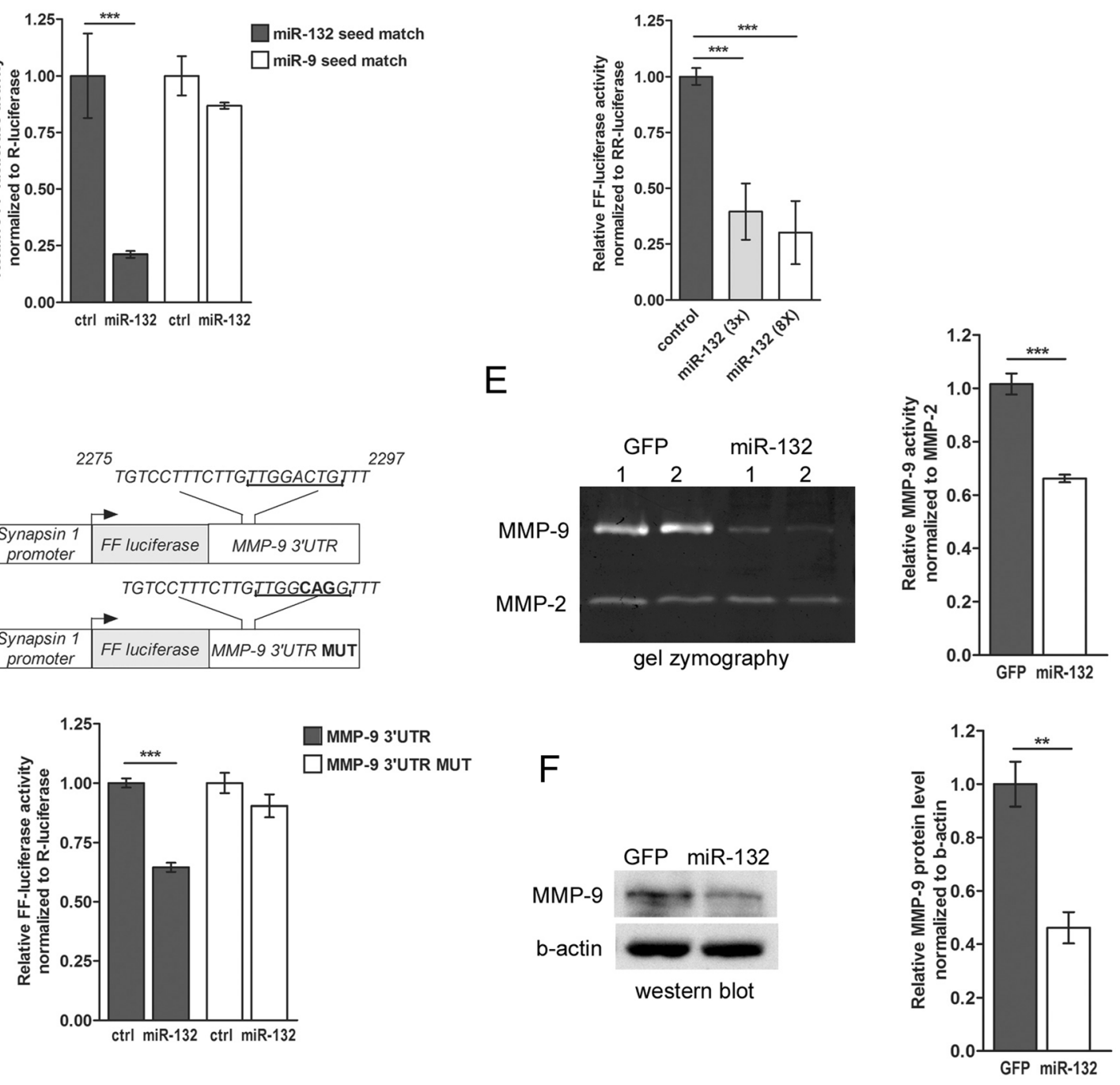

have observed a dose-dependent effect of miR-132 inhibition on luciferase activity (Fig. 2c). To characterize the exact miR132 binding site within the 3'UTR of MMP-9 transcript, the mutation was introduced into the putative miR-132 binding site (Fig. 2d). The cortical neurons in culture were transfected with the luciferase reporters together with the miR-132 overexpressing vector or EGFP expressing vector as a control. Overexpression of miR-132 in cortical neurons significantly

reduced the luciferase activity by about $30 \%$ compared to the control. Importantly, miR-132 failed to regulate the mutated MMP-9 3'UTR luciferase reporter, confirming the functionality of the predicted sequence within the 3'UTR of MMP-9 (Fig. 2d).

To validate if miR-132 can regulate the level of endogenous MMP-9 in neurons, we transfected primary cortical cultures with miR-132 precursor hairpin to overexpress the 
Fig. 2 miR-132 targets 3'UTR of MMP-9 mRNA and regulates the level of MMP-9 activity in neurons. a Alignment of the murine 3'UTR sequence of MMP-9 with miR-132. b miRNA expression and activity in neurons was validated on FF-luc reporters containing perfect-match miRNA target-sites (sensors) Error bars indicate SEM, $n=5$, $* * * p<0.001$ by Student's $t$ test. c Schematic representation of the luciferase reporter constructs - 3'UTR of MMP-9 mRNA was fused to the coding sequence of firefly luciferase $(F F-l u c)$. The construct was validated in HEK cells. Error bars indicate SEM, $n=5, * * * p<0.001$ by Student's $t$ test. d Schematic representation of the pSyn-Luc-3'UTRMMP9 luciferase reporter construct and its mutated version-the mutation was introduced in the putative miR-132 target site of MMP-9 3'UTR, four nucleotides were changed. miR-132 failed to regulate the mutated MMP-9 3'UTR luciferase reporter, suggesting that it binds to the predicted sequence. The FF-luc reporter constructs were cotransfected with plasmid expressing miR-132 in primary rat cortical neurons, along with a plasmid encoding Renilla reniformis luciferase $(R R-l u c)$ for normalization. Error bars indicate SEM, $n=5$, *** $p<0.001$ by Student's $t$ test. e Cortical neurons were electroporated with EGFP or miR-132 precursor hairpin to overexpress the mature microRNA. The level of MMP-9 and MMP-2 was assessed in the culture medium from transfected cells by gel zymography. The activities of MMP-9 and MMP2 were quantified by densitometry. Error bars indicate SEM, $n=5$, $* * * p<0.001$ by Student's $t$ test. f Western blot analysis with specific anti-MMP-9 and anti-beta-actin antibodies. The level of MMP-9 was quantified and normalized to beta-actin. Error bars indicate SEM, $n=3$, $* * p<0.01$ by Student's $t$ test

mature microRNA. MMP-9 is secreted by neurons in response to different types of synaptic stimulation; therefore, we measured its enzymatic activity in the cell culture medium using gel zymography. Overexpression of miR-132 reduced the level of secreted MMP-9 protein by about $40 \%$ when compared to the control neurons transfected with EGFP expressing vector (Fig. 2e). In the same culture media samples, the level of matrix metalloproteinase 2 (MMP-2) did not change, indicating that mir-132 specifically regulates endogenous MMP-9 in neurons. Western blot analysis with specific anti-MMP-9 antibody further confirmed the downregulation of MMP-9 protein by miR-132 in neurons overexpressing miR-132 (Fig. 2f).

\section{Activity-Dependent Translation of MMP-9 Involves miR-132}

Primary neurons in culture express spontaneous action potentials that activate the neuronal network and this can be responsible for the baseline expression level of miR-132. Therefore, we have checked whether the diminished neuronal activity will influence the level of endogenous miR-132. We transfected luciferase reporter construct containing the 3'UTR of MMP-9 mRNA and silenced the endogenous neuronal activity, by incubating the cells with a cocktail of inhibitors TTX (1 $\mu \mathrm{M})$, CNQX $(40 \mu \mathrm{M})$, APV $(100 \mu \mathrm{M})$, and nifedipine $(5 \mu \mathrm{M})$ for $10 \mathrm{~h}$. The reporter responded to the silencing of neuronal activity with the significant decrease of luciferase (Fig. 3a). This result suggests that the association of miR-132 with the 3'UTR of MMP-9 can be regulated by neuronal activity.
In the previous studies, we have shown that MMP-9 mRNA can associate with polyribosomal fractions isolated from stimulated synaptoneurosomes. Here, we investigated the polysomal distribution of endogenous miR-132 from control and DHPG-stimulated synaptoneurosomes. Synaptoneurosomes were incubated with $50 \mu \mathrm{M}$ DHPG for $20 \mathrm{~min}$, and the polyribosomes were separated by ultracentrifugation on linear sucrose gradient (Fig. 3b). Collected fractions were divided into five groups, which correspond to, from the top to the bottom of the gradient, free messenger ribonucleoprotein (mRNP) complexes; monosomal fraction; light polysomes; heavy polysomes, corresponding to the actively translating polyribosomal fraction; and RNA granules, the heavy fraction that sediments below the polysomes and contains RNA granules [35]. Equal amount of synthetic RNA (U6 snRNA-associated Sm-like protein-LSM from A. thaliana) was added to each fraction before RNA extraction for normalization. Total RNA was extracted from the gradient fractions and then analyzed by quantitative PCR. DHPG treatment resulted in the significant increase of miR-132 level in fraction 1 , representing $\mathrm{mRNPs}$, what can suggest its dissociation from MMP-9 mRNA which can be now translated and was shown to associate with polyribosomes in stimulated synaptoneurosomes.

\section{Overexpression of miR-132 in the Mature Neurons Regulates Dendritic Spine Structure by Direct Targeting of MMP-9 mRNA}

The increased level of MMP-9 secreted on dendritic spines has a pronounced effect on synaptic physiology and provoked changes in dendritic spine morphology (elongation of spines). The opposite dendritic spine shape changes were observed due to the miR-132 overexpression [18]. We have previously shown that in the mouse model of fragile X syndrome-Fmr1 $\mathrm{KO}$ mice - there is an increased synaptic translation of MMP9, which results in the higher activity of the enzyme on synapses and contributes to dendritic spine dysmorphologies [22]. Therefore, we used this experimental model of endogenous MMP-9 overexpression to observe the effect of miR-132 on dendritic spine morphology of mature neurons in culture. Hippocampal neurons from wt or Fmr1 KO mice were transfected at 14 days in vitro (DIV14) with plasmid for miR-132 overexpression or plasmid expressing EGFP. Three days after the transfection, neurons were fixed and dendritic spines were visualized and morphologically assessed (Fig. 4a-d). The spines were first sorted into three categories depending on their shape: these which displayed clear heads-mushroom spines, filopodia-like (thin), and small spines without heads (stubby). The parameters measured for long and stubby spines were total spine area, spine length, and spine width. For the mushroom spines, we analyzed spine head parameters - length, width , and area as well as total 
Fig. 3 Reduction of the synaptic activity inhibits translation of MMP-9 3'UTR luciferase reporter. a Luciferase gene activity in a reporter vector containing the wild-type 3' UTR of MMP-9 mRNA downstream of a luciferase gene in the control conditions and after the treatment with inhibitors. Error bars indicate SEM, $n=3$, $* * * p<0.001$ by Student's $t$ test. $\mathbf{b}$ The level of miR-132 on polisomal fractions isolated from synaptoneurosomes in control conditions and after gp1 mGluR stimulation with DHPG. Error bars indicate SEM, $n=5$, ${ }^{*} p<0.001$ by Mann-Whitney test

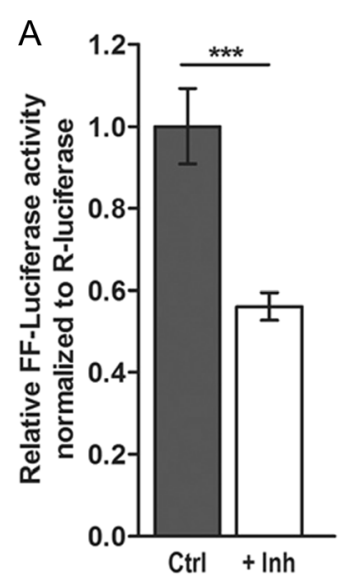

B

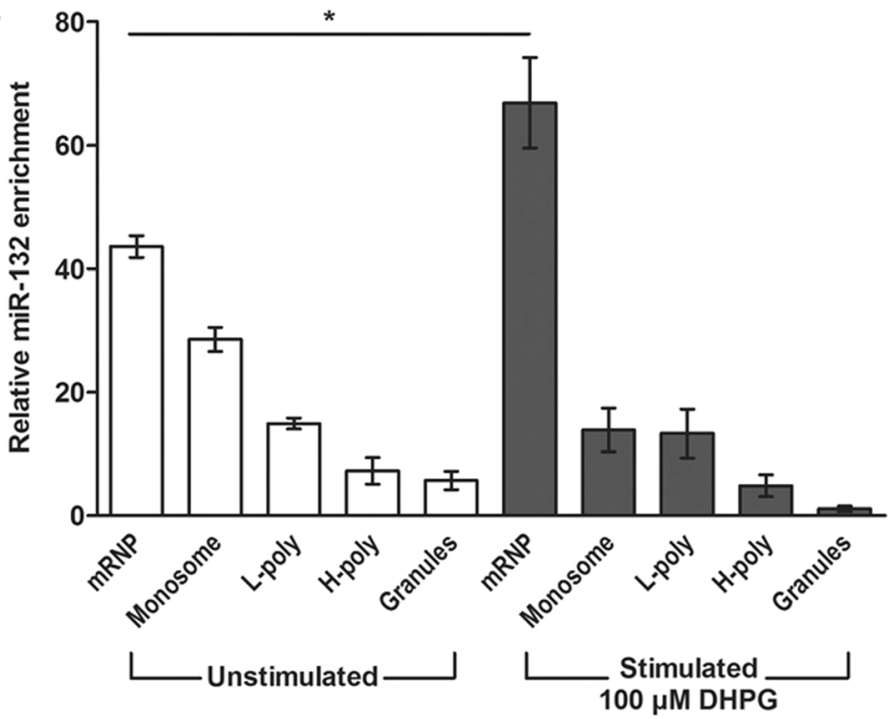

spine area. Overexpression of miR-132 in wt and Fmrl KO neurons resulted in spine head enlargement, when compared to the EGFP transfected controls (Fig. 4e-h). We have observed a significant increase in spine head area $(22 \%$ for wt and $15 \%$ for Fmr1 KO), spine head length (10\% for wt and $9 \%$ for Fmr1 KO), and spine head width (12\% for wt and $7 \%$ for Fmr1 KO) after overexpression of miR-132 in wt and Fmr1 KO neurons only for the spines classified as mushroom spines. The shape of thin and stubby spines was not affected by the overexpression of miR-132 (Figs. $4 i-n$ and 5). Significant change in spine shape was also observed between the genotypes-Fmr1 KO neurons had longer filopodia (medium length was increased for about $28 \%$ ), the stubby spines were thinner (for about $9 \%$ ), and for the mushroom spines the spine head width was reduced (for about $13 \%)(n=26$; total 6558 spines counted). This data suggest that overexpression of miR-132 in mature hippocampal neurons can regulate structural plasticity of dendritic spines through downregulation of matrix metalloproteinase 9 and may reverse the aberrant morphology of Fmr1 KO spines. We did not observe the significant changes in spine density between wt neurons and Fmr1 KO neurons transfected either with EGFP expressing vector or the one overexpressing miR-132. The average spine densities (spines $/ 10 \mu \mathrm{m}$ stretch of dendrite) were wt, $4( \pm 0.9$, $n=27)$; $\mathrm{wt}+$ miR-132, $4.7( \pm 1.2, n=27)$; FX, $4.9( \pm 1.4, n=27)$; and FX+miR-132, $4.3( \pm 1.1, n=27)$.

\section{Discussion}

In the present study, we provide evidence for the regulation of MMP-9 mRNA by miR-132 in neurons. Our results demonstrate a direct link between the two - not studied together before-important regulators of the synaptic plasticity that underlies learning and memory as well as major neuropsychiatric disorders.

Both miR-132 and MMP-9 are well-described regulators of synaptic plasticity. MMP-9 mRNA undergoes local synthesis at the synapse in response to neuronal stimulation [21] and is released to the extracellular space to regulate structural and functional spine remodeling. Direct influence of recombinant MMP-9 protein on dynamic spine changes has recently been reported [25, 36, 24]. For example, treatment of hippocampal neurons with autoactivating MMP-9 caused elongation and thinning of dendritic spines [24, 25], and the enlargement of dendritic spines in response to chemical long-term potentiation (cLTP) was blocked by MMP-9 inhibitors [37]. Also, the study of Bilousova et al. have demonstrated that a MMP-9 inhibitor-minocycline - promotes maturation of wt and Fmr1 KO dendritic spines both in cultures and in vivo. The beneficial effects of minocycline on dendritic spine morphology were also accompanied by changes in the behavioral performance of Fmr1 KO mice [25]. Finally, in the double Fmr1/ MMP-9 knockout mice, the immature phenotype of Fmr1 KO dendritic spines was rescued by genetic elimination of MMP9 activity [28]. Moreover, the reduction of exaggerated MMP9 mRNA translation in Fmrl(-/y) mice by the inhibition of translation initiation (reduction of eIF4E phosphorylation) also rescued the dendritic spine morphology defects [29]. The increased MMP-9 activity is observed in neurons as early as 10 min after the stimulation. Synaptic MMP-9 translation may allow for fast regulatory effect on spine morphology and receptor signaling. Our data strongly support a role for miR-132 in the regulation of activity-dependent synaptic translation of MMP-9. In the previous studies, miR-132 has been shown to bind and regulate the MMP-9 3'UTR during the development of the mammary glands in mice [38].

miR-132 was shown to regulate the expression of synaptic mRNAs involved in the modulation of synaptic excitability. 
Fig. 4 The effect of mir-132 overexpression on the dendritic spines morphology of wt and Fmr1 KO neurons. a-d

Hippocampal neurons isolated from wild-type or Fmr1 KO mice were transfected (DIV14+3) with EGFP- or miRNA-132

expressing vectors. Neurons were immunostained with anti-GFP antibody and imaged in the confocal microscope. e-n Dendritic spine morphology was analyzed, measures such as the spine area, head area, head length, and head width were quantified using the semi-automated spineMagic software for dendritic protrusions clustered into three categories - mushroom spines $(\mathbf{e}-\mathbf{h})$, thin spines $(\mathbf{i}-\mathbf{k})$, and stubby spines (1-n); 6550 spines were analyzed, $n=27$ for each experimental condition. Error bars indicate $\mathrm{SEM}, * p<0.05$; $* * * p<0.01 ; * * * p<0.001$ by Mann-Whitney test
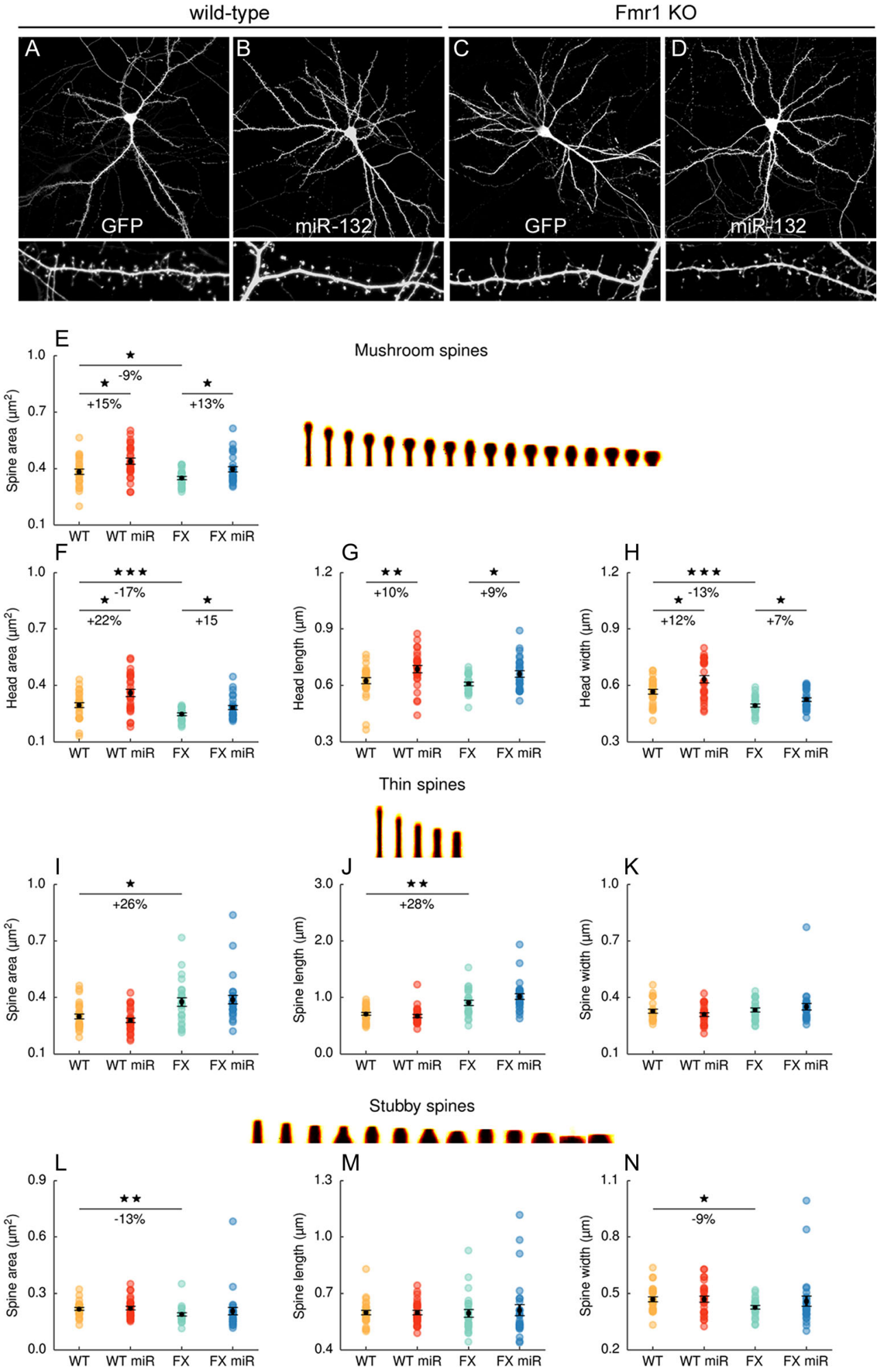

Impey and collaborators showed that miR-132-mediated inhibition of a Rho GTPase activator, p250GAP, activates the RAC1-PAK actin-remodeling pathway and thereby regulate activity-dependent spine plasticity in hippocampal neurons $[39,12]$. miR-132 can also inhibit the translation of methyl
CpG-binding protein $2(\mathrm{MeCP} 2)$ that in turn regulates $\mathrm{BDNF}$ expression. Downregulation of $\mathrm{MeCP} 2$ level during the postnatal period delays neuronal maturation and synapses formation [40], and opposite - its overexpression triggers dendrite and axon arborization [41, 42]. Another described target of 


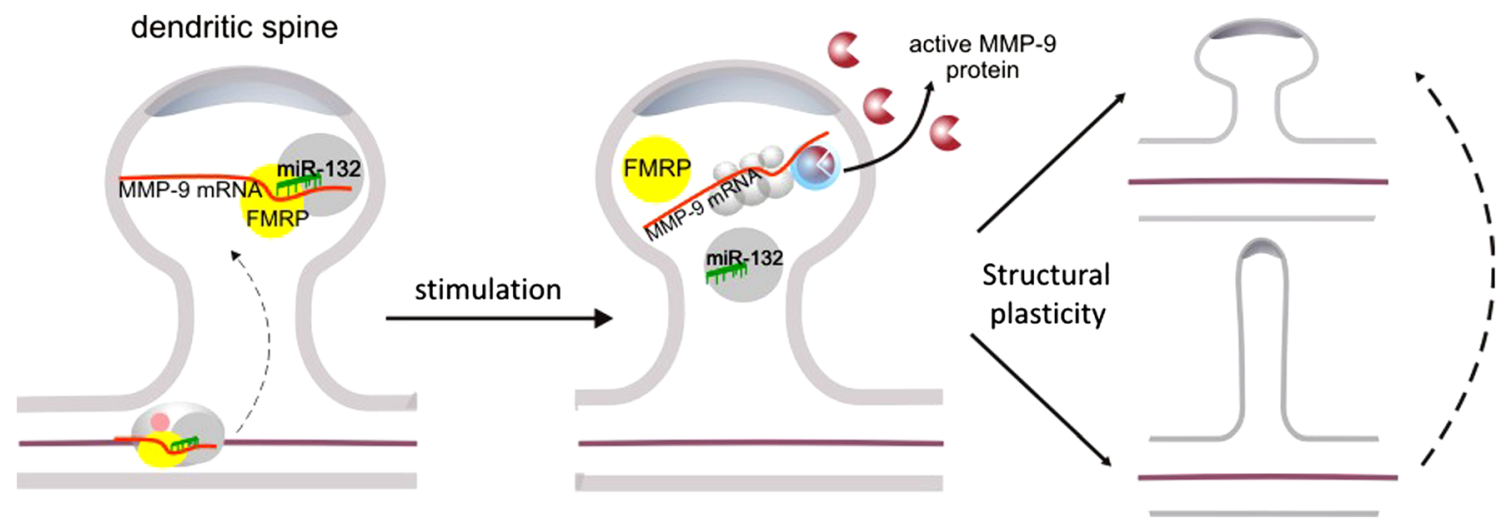

Fig. 5 Model depicting the role of miR-132 in the regulation of MMP-9 mRNA translation in response to synaptic stimulation at dendritic spines. FMRP is found in protein-RNA complex with MMP-9 mRNA and miR132. Based on the published studies $[45,18]$ and our own results, we

miR-132 is the deacetylase sirtuin 1 (SIRT1) [43]. SIRT1 was recently shown to enhance synaptic plasticity in a mouse hippocampus through a mechanism involving repression of miR134 , resulting in increased expression of BDNF and CREB [44]. The unique feature of MMP-9, the new miR-132 target that we describe here, is that it is secreted at the synapse to directly regulate the morphology of dendritic spines. Our discovery strengthens the importance of the activity-regulated miR-132 as a crucial controller of neuronal plasticity.

In neurons, the role of synaptic microRNAs is believed to be related to the repression of mRNA translation that can be alleviated due to the neuronal stimulation [45]. We observed that translational inhibition of MMP-9 mRNA by miR-132 was regulated by neuronal activity. The expression of luciferase from the reporter vector containing MMP-9 3'UTR was suppressed by the silencing of neuronal network activity of cultured neurons. A similar mechanism was observed for PSD-95 mRNA regulation by miR-125a where the PSD-95 mRNA translation was relieved by activation of gp1 mGluR signaling and dissociation of miR-125a [46]. MicroRNAs have also been shown to copurify with polysomes, what is probably related to their involvement in the repression of translation [47]. In our previous studies, we showed that MMP-9 mRNA is associated with actively translating polyribosomes in synaptoneurosomes and the in vitro stimulation of gp1 mGluR promotes its polysome assembly [22]. Herein, we show that a fraction of miR-132 is associated with polysomes in unstimulated synaptoneurosomes and the stimulation of gp1 mGluRs promotes the association of miR-132 with fraction 1 corresponding to mRNPs. These results suggest that miR-132 can reversibly regulate MMP-9 mRNA translation at synapses downstream of mGluR signaling. Many microRNAs were shown to co purify with polyribosomes in mammalian neurons [48]. In neurons, the local synthesis of synaptic proteins is induced by increased synaptic transmission. Therefore, it is possible that synaptic stimulation leads to hypothesize that activity-dependent release of MMP-9 protein at the synapse is regulated by miR-132 and FMRP, and leads to the structural changes of dendritic spines

the change in the polysome occupancy by specific microRNA and derepression of their target mRNAs. In the mouse model of mature microRNAs, depletion in neurons, the inactivation of the Dicerl gene responsible for microRNA maturation, leads to the progressive loss of a whole set of brain-specific miRNAs. Interestingly, the MMP-9 enzymatic activity was upregulated in the brain of mice with disruption of the Dicer1 gene in forebrain neurons (Dicer ${ }^{\text {CaMKCreERT2 }}$ [49]. This result further supports our hypothesis that microRNAs are responsible for MMP-9 mRNA translational repression.

Regulation of synaptic MMP-9 mRNA translation is crucial for its activity-dependent secretion that enables plastic changes of dendritic spine morphology. Previous studies have shown the effect of miR-132 on dendritic spine shape $[18,6$, 11]. Also MMP-9 was shown to regulate spine morphology in wt and Fmr1 KO mice [23-25].

In our study, the overexpression of miR-132 in neurons led to the increase in the dendritic spines heads; however, modulation of spine shape was more pronounced in case of wildtype cells than Fmr1 KO neurons. Interestingly, the width of the spine head increased for about $12 \%$ and the spine head area for $22 \%$ for wild-type dendritic spines, while for the Fmr1 KO neurons it was 7 and $15 \%$ respectively. This would implicate that the FMRP has an additional regulatory effect on the miR-132-MMP-9 interaction. An example of such regulatory mechanism is PSD-95 mRNA shown to be regulated by miR-125b and FMRP activity-dependent phosphorylation [45]. Further study is needed to reveal this question for MMP-9 mRNA and miR-132.

In aggregate, in the present study, we provide evidence that neuronal stimulation-driven rapid MMP-9 protein synthesis can be an effect of miR-132-dependent derepression of MMP-9 mRNA. Our findings provide an insight into a molecular mechanism that involves miR-132-regulated MMP-9 expression in neurons and their function for structural plasticity of dendritic spines. 
Acknowledgments This work was supported by a grant from the Polish National Science Center (Harmonia 2013/08/M/NZ4/00650) for MD, Preludium from the Polish National Science Center (2012/07/N/NZ3/ 01509) for JM, and Foundation for Polish Science, FNP TEAM for LK.

Open AccessThis article is distributed under the terms of the Creative Commons Attribution 4.0 International License (http:// creativecommons.org/licenses/by/4.0/), which permits unrestricted use, distribution, and reproduction in any medium, provided you give appropriate credit to the original author(s) and the source, provide a link to the Creative Commons license, and indicate if changes were made.

\section{References}

1. Bartel DP (2009) MicroRNAs: target recognition and regulatory functions. Cell 136(2):215-233

2. Huntzinger E, Izaurralde E (2011) Gene silencing by microRNAs: contributions of translational repression and mRNA decay. Nat Rev Genet 12(2):99-110

3. Fabian MR, Sonenberg N, Filipowicz W (2010) Regulation of mRNA translation and stability by microRNAs. Annu Rev Biochem 79:351-379

4. Bicker S, Lackinger M, Weiss K, Schratt G (2014) MicroRNA-132, -134, and -138: a microRNA troika rules in neuronal dendrites. Cell Mol Life Sci CMLS 71(20):3987-4005

5. Nudelman AS, DiRocco DP, Lambert TJ, Garelick MG, Le J, Nathanson NM, Storm DR (2010) Neuronal activity rapidly induces transcription of the CREB-regulated microRNA-132, in vivo. Hippocampus 20(4):492-498

6. Mellios N, Sugihara H, Castro J, Banerjee A, Le C, Kumar A, Crawford B, Strathmann J et al (2011) miR-132, an experiencedependent microRNA, is essential for visual cortex plasticity. Nat Neurosci 14(10):1240-1242

7. Tognini P, Putignano E, Coatti A, Pizzorusso T (2011) Experiencedependent expression of miR-132 regulates ocular dominance plasticity. Nat Neurosci 14(10):1237-1239

8. Wibrand K, Pai B, Siripornmongcolchai T, Bittins M, Berentsen B, Ofte ML, Weigel A, Skaftnesmo KO et al (2012) MicroRNA regulation of the synaptic plasticity-related gene Arc. PLoS One 7(7): e41688

9. Remenyi J, Hunter CJ, Cole C, Ando H, Impey S, Monk CE, Martin KJ, Barton GJ et al (2010) Regulation of the miR-212/132 locus by MSK1 and CREB in response to neurotrophins. Biochem J 428(2):281-291

10. Pathania M, Torres-Reveron J, Yan L, Kimura T, Lin TV, Gordon V, Teng ZQ, Zhao X et al (2012) miR-132 enhances dendritic morphogenesis, spine density, synaptic integration, and survival of newborn olfactory bulb neurons. PloS One 7(5): e38174

11. Luikart BW, Bensen AL, Washburn EK, Perederiy JV, Su KG, Li Y, Kernie SG, Parada LF et al (2011) miR-132 mediates the integration of newborn neurons into the adult dentate gyrus. PloS One 6(5): e19077

12. Wayman GA, Davare M, Ando H, Fortin D, Varlamova O, Cheng HY, Marks D, Obrietan K et al (2008) An activity-regulated microRNA controls dendritic plasticity by down-regulating p250GAP. Proc Natl Acad Sci U S A 105(26):9093-9098

13. Remenyi J, van den Bosch MW, Palygin O, Mistry RB, McKenzie C, Macdonald A, Hutvagner G, Arthur JS et al (2013) miR-132/212 knockout mice reveal roles for these miRNAs in regulating cortical synaptic transmission and plasticity. PloS One 8(4):e62509

14. Vo NK, Cambronne XA, Goodman RH (2010) MicroRNA pathways in neural development and plasticity. Curr Opin Neurobiol 20(4):457-465
15. Siegel G, Saba R, Schratt G (2011) microRNAs in neurons: manifold regulatory roles at the synapse. Curr Opin Genet Dev 21(4): 491-497

16. Wanet A, Tacheny A, Arnould T, Renard P (2012) miR-212/132 expression and functions: within and beyond the neuronal compartment. Nucleic Acids Res 40(11):4742-4753

17. Magill ST, Cambronne XA, Luikart BW, Lioy DT, Leighton BH, Westbrook GL, Mandel G, Goodman RH (2010) microRNA-132 regulates dendritic growth and arborization of newborn neurons in the adult hippocampus. Proc Natl Acad Sci U S A 107(47):2038220387

18. Edbauer D, Neilson JR, Foster KA, Wang CF, Seeburg DP, Batterton MN, Tada T, Dolan BM et al (2010) Regulation of synaptic structure and function by FMRP-associated microRNAs miR$125 \mathrm{~b}$ and miR-132. Neuron 65(3):373-384

19. Huntley GW (2012) Synaptic circuit remodelling by matrix metalloproteinases in health and disease. Nat Rev Neurosci 13(11):74357

20. Rivera S, Khrestchatisky M, Kaczmarek L, Rosenberg GA, Jaworski DM (2010) Metzincin proteases and their inhibitors: foes or friends in nervous system physiology? J Neurosci 30:1533715357

21. Dziembowska M, Milek J, Janusz A, Rejmak E, Romanowska E, Gorkiewicz T, Tiron A, Bramham CR et al (2012) Activitydependent local translation of matrix metalloproteinase-9. J Neurosci Off J Soc Neurosci 32(42):14538-14547

22. Janusz A, Milek J, Perycz M, Pacini L, Bagni C, Kaczmarek L, Dziembowska M (2013) The Fragile X mental retardation protein regulates matrix metalloproteinase $9 \mathrm{mRNA}$ at synapses. J Neurosci Off J Soc Neurosci 33(46):18234-18241

23. Dityatev A, Schachner M, Sonderegger P (2010) The dual role of the extracellular matrix in synaptic plasticity and homeostasis. Nat Rev Neurosci 11(11):735-746

24. Michaluk P, Wawrzyniak M, Alot P, Szczot M, Wyrembek P, Mercik K, Medvedev N, Wilczek E et al (2011) Influence of matrix metalloproteinase MMP-9 on dendritic spine morphology. J Cell Sci 124:3369-3380

25. Bilousova TV, Dansie L, Ngo M, Aye J, Charles JR, Ethell DW, Ethell IM (2009) Minocycline promotes dendritic spine maturation and improves behavioural performance in the fragile $\mathrm{X}$ mouse model. J Med Genet 46(2):94-102. doi:10.1136/jmg.2008.061796

26. Rudelli RD, Brown WT, Wisniewski K, Jenkins EC, LaureKamionowska M, Connell F, Wisniewski HM (1985) Adult fragile $\mathrm{X}$ syndrome. Clinico-neuropathologic findings. Acta Neuropathol 67(3-4):289-295

27. Comery TA, Harris JB, Willems PJ, Oostra BA, Irwin SA, Weiler IJ, Greenough WT (1997) Abnormal dendritic spines in fragile X knockout mice: maturation and pruning deficits. Proc Natl Acad Sci U S A 94(10):5401-5404

28. Sidhu H, Dansie LE, Hickmott PW, Ethell DW, Ethell IM (2014) Genetic removal of matrix metalloproteinase 9 rescues the symptoms of fragile X syndrome in a mouse model. J Neurosci Off J Soc Neurosci 34(30):9867-9879

29. Gkogkas CG, Khoutorsky A, Cao R, Jafarnejad SM, PragerKhoutorsky M, Giannakas N, Kaminari A, Fragkouli A et al (2014) Pharmacogenetic inhibition of eIF4E-dependent Mmp9 mRNA translation reverses fragile $\mathrm{X}$ syndrome-like phenotypes. Cell Rep 9(5):1742-1755

30. Ruszczycki B, Szepesi Z, Wilczynski GM, Bijata M, Kalita K, Kaczmarek L, Wlodarczyk J (2012) Sampling issues in quantitative analysis of dendritic spines morphology. BMC Bioinforma 13:213

31. Te O (2007) Python for scientific computing. Comput Sci Eng 9: $10-20$

32. Pérez FGB (2007) IPython: a system for interactive scientific computing. Comput Sci Eng 9:21-29 
33. Jd H (2007) Matplotlib: a 2D graphics environment. Comput Sci Eng 9:90-95

34. Zalfa F, Giorgi M, Primerano B, Moro A, Di Penta A, Reis S, Oostra B, Bagni C (2003) The fragile X syndrome protein FMRP associates with $\mathrm{BC} 1 \mathrm{RNA}$ and regulates the translation of specific mRNAs at synapses. Cell 112(3):317-327

35. Krichevsky AM, Kosik KS (2001) Neuronal RNA granules: a link between RNA localization and stimulation-dependent translation. Neuron 32(4):683-696

36. Bozdagi O, Nagy V, Kwei KT, Huntley GW (2007) In vivo roles for matrix metalloproteinase-9 in mature hippocampal synaptic physiology and plasticity. J Neurophysiol 98(1):334-344

37. Szepesi Z, Bijata M, Ruszczycki B, Kaczmarek L, Wlodarczyk J (2013) Matrix metalloproteinases regulate the formation of dendritic spine head protrusions during chemically induced long-term potentiation. PLoS One 8(5):e63314

38. Ucar A, Vafaizadeh V, Jarry H, Fiedler J, Klemmt PA, Thum T, Groner B, Chowdhury K (2010) miR-212 and miR-132 are required for epithelial stromal interactions necessary for mouse mammary gland development. Nat Genet 42(12):1101-1108

39. Impey S, Davare M, Lesiak A, Fortin D, Ando H, Varlamova O, Obrietan K, Soderling TR et al (2010) An activity-induced microRNA controls dendritic spine formation by regulating Rac1PAK signaling. Mol Cell Neurosci 43(1):146-156

40. Fukuda T, Itoh M, Ichikawa T, Washiyama K, Goto Y (2005) Delayed maturation of neuronal architecture and synaptogenesis in cerebral cortex of Mecp2-deficient mice. J Neuropathol Exp Neurol 64(6):537-544

41. Klein ME, Lioy DT, Ma L, Impey S, Mandel G, Goodman RH (2007) Homeostatic regulation of MeCP2 expression by a CREBinduced microRNA. Nat Neurosci 10(12):1513-1514
42. Jugloff DG, Jung BP, Purushotham D, Logan R, Eubanks JH (2005) Increased dendritic complexity and axonal length in cultured mouse cortical neurons overexpressing methyl-CpG-binding protein MeCP2. Neurobiol Dis 19(1-2):18-27

43. Strum JC, Johnson JH, Ward J, Xie H, Feild J, Hester A, Alford A, Waters KM (2009) MicroRNA 132 regulates nutritional stressinduced chemokine production through repression of SirT1. Mol Endocrinol 23(11):1876-1884

44. Gao J, Wang WY, Mao YW, Graff J, Guan JS, Pan L, Mak G, Kim D et al (2010) A novel pathway regulates memory and plasticity via SIRT1 and miR-134. Nature 466(7310):1105-1109

45. Swanger SA, Bassell GJ (2013) Dendritic protein synthesis in the normal and diseased brain. Neuroscience 232:106-127

46. Muddashetty RS, Nalavadi VC, Gross C, Yao X, Xing L, Laur O, Warren ST, Bassell GJ (2011) Reversible inhibition of PSD-95 mRNA translation by miR-125a, FMRP phosphorylation, and mGluR signaling. Mol Cell 42(5):673-688

47. Molotski N, Soen Y (2012) Differential association of microRNAs with polysomes reflects distinct strengths of interactions with their mRNA targets. RNA 18(9):1612-1623

48. Kim J, Krichevsky A, Grad Y, Hayes GD, Kosik KS, Church GM, Ruvkun G (2004) Identification of many microRNAs that copurify with polyribosomes in mammalian neurons. Proc Natl Acad Sci U S A 101(1):360-365

49. Konopka W, Kiryk A, Novak M, Herwerth M, Parkitna JR, Wawrzyniak M, Kowarsch A, Michaluk P et al (2010) MicroRNA loss enhances learning and memory in mice. J Neurosci Off J Soc Neurosci 30(44):14835-14842 